\section{Hábitos orais de sucção: estudo piloto em população de baixa renda}

\author{
Valdinês G.S. Cavassani', Sonize G. Ribeiro', \\ Nair K. Nemr' ${ }^{2}$ Ana M.Greco3 , Juliana Köhle, \\ Carlos N. Lehn
}

Resumo

$\mathbf{H}$ ábitos orais de sucção têm sido fonte de estudo pelos danos que podem causar em toda morfologia e função do sistema estomatognático, sendo comumente iniciados e observados na infância. Fatores emocionais e nutricionais podem contribuir para a manifestação desses hábitos. Objetivo: Verificar alterações fonoaudiológicas, odontológicas e otorrinolaringológicas em crianças de baixa renda portadoras de hábitos orais de sucção. Forma de Estudo: Clínico retrospectivo não randomizado. Material e Método: Foram avaliadas nove crianças ( 01 menino e 08 meninas) com idades entre 5 a 9 anos durante o I Mutirão da Comunicação realizado no Hosphel, São Paulo, no dia 27 de outubro de 2001. Resultados: O distúrbio fonoaudiológico mais comum foi o articulatório (55,56\%), seguido pelo distúrbio de motricidade oral (33,33\%). A mordida aberta esteve presente em 8 casos $(88,89 \%)$. Com relação ao tipo de respiração apresentou-se com 7 casos com respiração bucal $(77,78 \%)$. Conclusões: Os hábitos orais foram capazes de promover alterações fonoaudiológicas, odontológicas e otorrinolaringológicas. Medidas de promoção de saúde devem ser lançadas para crianças de baixa renda visando eliminar os fatores responsáveis pela origem dos hábitos orais viciosos de sucção, tais como: alimentação e educação. Além de medidas de tratamento multidisciplinar, devem ser estabelecidas fonoaudiologia, ortodontia, otorrinolaringologia em saúde pública, solucionando o paradigma das alterações decorrentes desses hábitos comumente perceptíveis na infância.

\section{Suction oral habits: initial study in low income population}

Palavras-chave: hábitos orais de sucção, determinantes sócioeconômicos, má oclusão, alterações fonoaudiológicas.

Key words: suction oral habits, socioeconomic determinants, malocclusion, phonoaudiological disturbances.

Uuction oral habits have been observed in childhood due to emotional and nutritional factors, leading to dental and speech and voice consequences. Objective: The purpose of this study was the observation of dental and speech and articulation abnormalities in low-income children with suction oral habits. Study Design: Retrospective clinical non randomized study. Material and Method: Nine Brazilian children ( 8 girls and 1 boy) were examined in "I Mutirão da Comunicação" at Heliópolis Hospital, Hosphel/São Paulo, Brazil, on October 27, 2001. Results: The most common speech and voice disorder detected was articulation impairment $(55.56 \%)$, followed by oral motor disorder (33.33\%). Open bite was detected in eight cases (88.89\%). In seven cases (77.78\%) we observed mouth breathing. Conclusions: Speech and articulation and dental disorders are normally detected in children with suction oral habits. The importance of interrelating dental, speech and otorhinolaryngology abnormalities in association with public health programs must be established for the low-income population.

\footnotetext{
'Mestranda pelo Curso de Pós-Graduação em Ciências da Saúde do Hospital Heliópolis - Hosphel, São Paulo.

${ }^{2}$ Docentes do Curso de Pós-Graduação em Ciências da Saúde do Hospital Heliópolis - Hosphel, São Paulo.

${ }^{3}$ Fonoaudiólogas do Hospital Heliópolis - Hosphel, São Paulo.

${ }^{4}$ Chefe do Departamento de Cirurgia de Cabeça e Pescoço e Otorrinolaringologia do Hospital Heliópolis - Hosphel, São Paulo.

Trabalho realizado no Hospital Heliópolis - Hosphel, São Paulo, SP.

Endereço para correspondência: Valdinês Gonçalves dos Santos Cavassani - Rua José Patrício, 43 Rudge Ramos São Bernardo do Campo SP 09601-010 Tel/fax: (0xx11) 4368-9666 - E-mail: hmpsa_comercial@ig.com.br

Artigo recebido em 10 de setembro de 2002. Artigo aceito em 29 de novembro de 2002.
} 


\section{INTRODUÇÃO}

Hábitos orais têm sido alvo de grande interesse dos profissionais de saúde ligados à área de motricidade. A motricidade oral engloba os aspectos relacionados à ação de grupos musculares nas movimentações, nas características morfológicas e na descrição de processos funcionais como articulação de fonemas, sucção, mastigação e deglutição em seus respectivos padrões normais e patológicos ${ }^{1}$. Isto porque eles estão intrinsecamente ligados à postura, mobilidade, tonicidade e sensibilidade dos órgãos fonoarticulatórios. Estas são assim denominados por serem responsáveis pela articulação de fonemas e pela fase oral da deglutição. Pela complexidade anatômica dessas estruturas musculares e por estarem tão intimamente ligadas, qualquer alteração pode ocasionar distúrbios, tanto fonoaudiológicos como odontológicos, cujas seqüelas afetam a parte funcional e estética de maneira significativa.

Os hábitos são padrões de contração muscular aprendidos, alguns deles servindo como fatores etiológicos das maloclusões de caráter muscular, esquelética ou dentária.

A avaliação comportamental do desenvolvimento motor oral deve ser realizada a fim de evidenciar as dificuldades e facilidades da criança nas situações da mastigação, sucção, deglutição, respiração e postural global. Uma vez observados hábitos orais viciosos, o sistema estomatognático pode sofrer disfunções significativas em função da freqüência, intensidade e duração dos mesmos².

Alguns dos fatores etiológicos responsáveis por hábitos orais incluem: conflitos familiares, pressão escolar, o "stress" das grandes cidades, irritações associadas com a erupção dental, interferências oclusais, obstrução respiratória, má postura e outros fatores emocionais ${ }^{3}$. Há, ainda, causas associadas a problemas da adenóide, nas tonsilas ou no próprio nariz (desvio do septo e hipertrofia de conchas). Estes quadros podem ter como conseqüência a respiração bucal, que interfere na tonicidade, postura, mobilidade e sensibilidade de órgãos fonoarticulatórios podendo gerar hábitos orais viciosos. É imprescindivel que a avaliação otorrinolaringológica seja realizada sempre que estão presentes os hábitos orais viciosos para que sejam descartados fatores anatomo-funcionais como etiologia do quadro clínico presente

As conseqüências das alterações otorrinolaringológicas podem promover alterações de oclusão ou mordida, tendo indicação de correção ortodôntica, associada à fonoterapia, pois a correção ortodôntica depende de reeducação postural, sem a qual o resultado pode ficar prejudicado 5 .

Os hábitos orais podem ser influenciados, assim como outros comportamentos, por alguns fatores sociais, como emprego da mãe que colabora na renda familiar de forma parcial ou total, inicialmente pelo padrão de aleitamento materno, dificuldade de acesso aos serviços odontológicos, algumas doenças respiratórias e problemas de fala, entre outros $^{6}$. Realizado um estudo com 357 crianças, na faixa etária de 3 a 5 anos da cidade de Belo Horizonte, focando a associação entre aleitamento, hábitos bucais e maloclusões, constatou-se que houve associação do aleitamento natural com a não instalação de hábitos orais viciosos em crianças que foram amamentadas no mínimo por seis meses, e a associação de hábitos bucais com maloclusões foi significante ${ }^{7}$. Estas variáveis devem ser melhor avaliadas, de forma a estabelecer como os fatores sócio-econômicos podem influenciar na má oclusão através de hábitos orais, fatores psicológicos e padrões de doenças gerais.

Os hábitos orais viciosos mais freqüentes são: sucção de lábio, dedo, bochecha ou de objetos e podem provocar alterações na arcada dentária e na mordida, hipotonia de órgãos fonoarticulatórios, respiração bucal, onicofagia e outros. A sucção, seja de dedo, de chupeta ou qualquer outro objeto tem sido fonte de estudo, pelo fato de ser tão freqüente e pelos danos que pode causar. Os hábitos de sucção trazem conseqüências importantes na morfologia do palato duro, alterações de posicionamentos dentais, movimentação da língua, com alterações musculares periorais e fonoarticulatórias, tendo maior risco de desenvolvimento de mordida aberta e distúrbios de motricidade oral ${ }^{1,8}$. Os hábitos orais viciosos decorrem da necessidade de suprir carências afetivas, geralmente transmitem sensação de segurança e conforto, podendo ser feito uso de elementos nutritivos e não nutritivos. A sucção é a primeira atividade muscular coordenada da criança sendo um fenômeno diretamente ligado à deglutição, também percebida antes do nascimento, sob a forma de contrações bucais ou outras respostas reflexas. A sucção é considerada um hábito nutritivo até os 3 anos de idade e vicioso após essa idade ${ }^{9,10,11,12}$. Vários estudos epidemiológicos foram feitos para avaliar a incidência de hábitos de sucção em crianças, obtendo-se cifras de 54,60\% ( 3 a 5 anos), em sua maioria respiradores bucais ${ }^{13}$. Outros estudos com crianças com idades entre 7 e 10 anos, de ambos os sexos, concluíram que o hábito persistente de sucção de chupeta não teve influência sobre as características esqueléticas. Entretanto, promoveram uma redução do ângulo interincisal, principalmente pela inclinação vestibular dos incisivos superiores e uma redução da altura dento-alveolar no seu nível, o que também implicou em aumento da sobressaliência e redução da sobremordida, porém não foram capazes de alterar permanentemente as medidas esqueléticas faciais ${ }^{14}$. Outros autores ${ }^{15}$, avaliando crianças com idades entre 2 e 6 anos, concluíram que hábitos orais de sucção de chupeta ou de dedo foram capazes de causar uma maior incidência de anomalias na oclusão dentária decídua. Um outro aspecto relacionado ao hábito de sucção é a possibilidade de desenvolvimento de estreitamento maxilar, provocando a mordida aberta anterior e mordida cruzada posterior $^{16,7}$. Quanto aos distúrbios fonoaudiológicos em portadores de hábitos de sucção de dedo, com idades entre 4 e 7 anos, observou-se que todas as crianças apresentavam distorções nos fonemas /s/ e /f/, fato este 
relacionado com a presença de mordida aberta provocada pelo hábito vicioso ${ }^{17}$. Todavia, um estudo longitudinal com crianças de 3 anos acompanhadas até os 21 anos de idade revelou que se o hábito fosse interrompido precocemente, os efeitos sobre a oclusão eram transitórios, embora nenhuma das crianças que abandonaram os hábitos viciosos após os 6 anos de idade apresentou oclusão normal aos 12 anos de idade $^{15}$, justificando a proposta que o tratamento anterior aos 6 anos de idade seja a remoção do hábito oral vicioso e após essa idade haja intervenção mecânica ortodôntica.

Os distúrbios articulatórios podem estar direta ou indiretamente relacionadas aos hábitos viciosos orais de sucção, sendo explicados a partir de alterações presentes de formas isoladas ou associadas como: omissão, substituição, distorção, adição e transposição de fonemas, sendo consideradas patológicas quando presentes a partir de 4 anos de idade ${ }^{18}$

Vários estudos têm sido realizados relacionando causas e efeitos dos hábitos orais viciosos de sucção, também referidos como deletérios ou prejudiciais ${ }^{2,5,9,10,20,21}$. Percebemos, desta forma, a importância da inter-relação que envolve obrigatoriamente as áreas de Fonoaudiologia, de Odontologia e de Otorrinolaringologia e a necessidade de estudos que tratem do tema e reforcem esta interdisciplinariedade no tratamento dos hábitos viciosos orais de sucção.

Este estudo teve por objetivo verificar as alterações fonoaudiológicas e odontológicas, e as possíveis correlações com aspectos otorrinolaringológicos, em crianças portadoras de hábito oral vicioso de sucção, a partir de um evento de promoção de saúde em que, preventivamente, foi oferecido à população de baixa renda da cidade de São Paulo um dia de triagem fonoaudiológica e inspeção bucal gratuitos.

\section{MATERIAL E MÉTODO}

A amostra foi constituída por 9 crianças brasileiras de ambos os sexos (1 menino e 8 meninas), com idades entre 5 e 9 anos, com hábitos orais viciosos de sucção, selecionadas no I Mutirão da Comunicação. O evento foi realizado no Hospital Heliópolis-SP no dia 27 de outubro de 2001 e o principal objetivo foi de conscientizar a população sobre os distúrbios da comunicação que podem ser evitados e tratados logo que surgirem os primeiros sinais de alterações da fala, vOz, respiração, mastigação, linguagem oral e escrita.

Inicialmente, os pacientes assistiam a um vídeo apresentado por uma dentista e uma fonoaudióloga, os quais falavam sobre as fases do desenvolvimento normal, do nascimento à terceira idade. A partir daí, passamos por prétriagem fonoaudiológica na qual, ao ser detectada qualquer alteração, o indivíduo era orientado a aguardar para então ser submetido às avaliações fonoaudiológicas e inspeções odontológicas.

Os dados foram coletados a partir de avaliações padronizadas e aplicadas por uma equipe de 7 fonoaudiólogos e 6 odontólogos, sendo que as respostas foram fornecidas pelos responsáveis e complementadas pelos exames profissionais realizados.

Foram atendidos 156 pacientes, com um total de 252 procedimentos realizados ( 156 triagens fonoaudiológicas, 50 inspeções bucais e 46 triagens audiológicas) e 109 encaminhamentos foram realizados (77 para Fonoterapia, 25 para Otorrinolaringologia, 02 para Psiquiatria/Psicologia, 02 para Neurologia, 02 para Cirurgia de Cabeça e Pescoço, 01 para Oftalmologia e Odontologia).

Coletamos os dados referentes a: sexo, idade, tipo e modo respiratório (nasal, bucal e misto), distúrbio fonoaudiológico (disfluência, alterações da motricidade oral, distúrbio articulatório), tipo de mordida (normal, aberta, cruzamento unilateral e sobremordida).

Devido ao tamanho da amostra não foi realizado teste estatístico, sendo apresentada nos resultados a descrição dos dados.

O Termo de Consentimento Livre e Esclarecido foi apresentado aos pais e/ou responsáveis que autorizaram a pesquisa, tendo sido aprovado pelo Comitê de Ética em Pesquisa do Hospital Heliópolis - São Paulo.

\section{RESULTADOS}

Nossa amostra é constituída de 9 crianças (1 menino e 8 meninas) com idades variando de 5 a 9 anos (duas crianças com 5 anos, uma criança com 6 anos, duas crianças com 7 anos, três crianças com 8 anos e uma criança com 9 anos). Quanto ao tipo de respiração, 7 casos apresentaram respiração predominante bucal (77,78\%), 1 caso respiração mista $(11,11 \%)$ e 1 caso normal $(11,11 \%)$.

Verificamos a ocorrência de distúrbios fonoaudiológicos e detectamos 5 casos com distúrbio articulatório (55,56\%), 3 casos com distúrbio de motricidade oral $(33,33 \%)$ e 1 caso com ausência de patologia fonoaudiológica $(11,11 \%)$. Consideramos distúrbio da motricidade as alterações de tonicidade, postura, mobilidade e/ou sensibilidade de órgãos fonoarticulatórios (Gráfico 1), e detectamos, ainda, 8 casos com mordida aberta (88,89\%), 1 caso de mordida normal, havendo nítida correlação da mordida aberta com o hábito de sucção (Gráfico 2).

\section{DISCUSSÃO}

O Hospital Heliópolis, situado em São Paulo, apresenta nos seus arredores cerca de 90 mil moradores de baixa renda. A idealização do I Mutirão da Comunicação foi baseada na necessidade crescente dessa população em terapêuticas especializadas e abordagem de medidas preventivas, através de palestras de conscientização nas diferentes especialidades: Fonoaudiologia, Odontologia e Otorrinolaringologia. Não havendo no serviço público a especialidade odontológica ortodôntica para efetivamente atuar nas correções mecânicas 


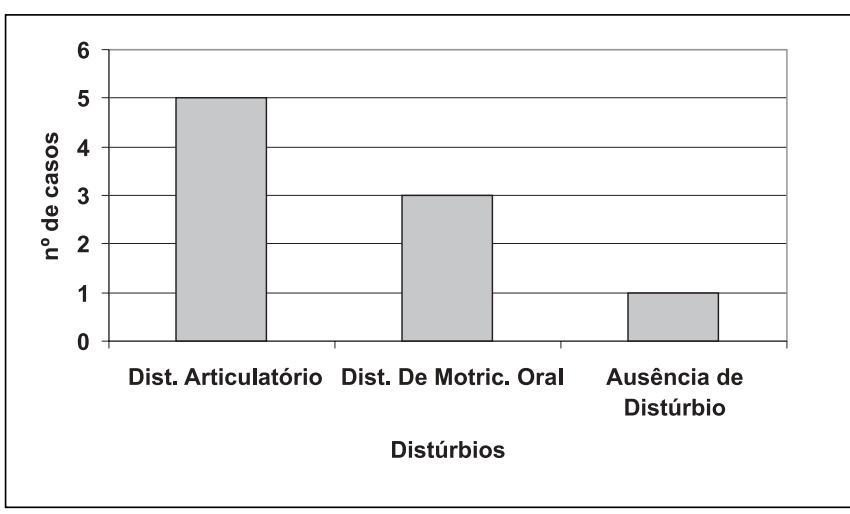

Gráfico 1. Distribuição da Ocorrência dos Distúrbios Fonoaudiológicos

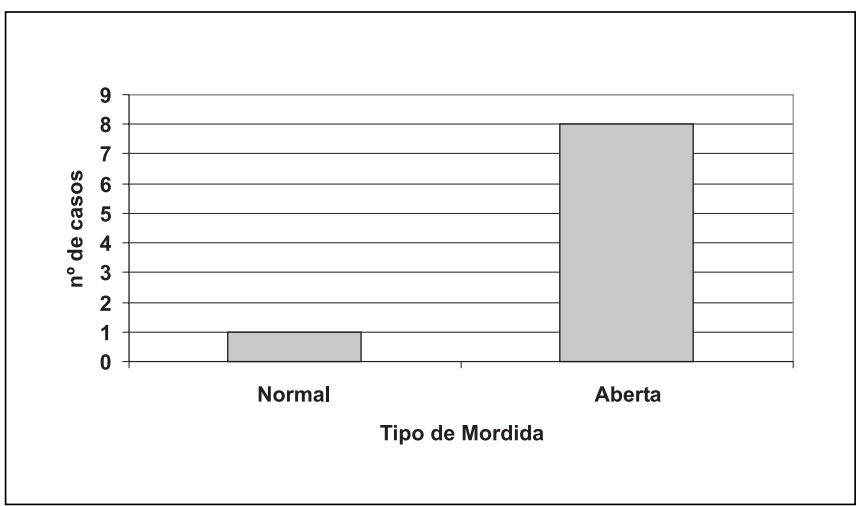

Gráfico 2. Distribuição quanto ao tipo de mordida

das alterações de mordida observadas nos sujeitos da pesquisa realizada (mordida aberta), não foi possível realizar estes encaminhamentos. Salientamos a necessidade da incorporação desta especialidade para que a população de baixa renda tenha acesso à essa terapêutica corretiva ortodôntica, assim tendo a sua estética e função restabelecidas e a qualidade de vida melhorada. O conceito de promoção de saúde foi definido na Carta de Ottawa, documento elaborado na I Conferência Internacional de Promoção da Saúde, em novembro de 1986, como sendo "o processo de capacitação da comunidade para atuar na melhoria da sua qualidade de vida e saúde, incluindo uma maior participação no controle deste processo". O documento menciona, ainda, uma série de condições e recursos necessários para se ter saúde, tais como: paz, habitação, educação, alimentação, renda, meio ambiente, justiça social e eqüidade. A partir desse documento a atuação das especialidades de ortodontia, de fonoaudiologia e de otorrinolaringologia) devem estar disponíveis para a população carente com acesso gratuito em serviços públicos.

O tipo de respiração foi um aspecto que nos chamou a atenção na pesquisa, pois obtivemos $77,78 \%$ dos casos com respiração predominantemente bucal. Segundo a literatura, as causas da respiração bucal envolvem: obstruções das vias aéreas superiores, desvios do septo nasal, inflamação da membrana basal, cornetos nasais hipertrofiados, tonsila faríngea hipertrofiada entre outros ${ }^{2,22}$. Notamos nos casos da pesquisa, que houve alteração morfológica do palato, com aprofundamento. Estas crianças apresentavam rosto alongado e estreito, olhos caídos, lábios entreabertos, hipotônicos e ressecados, sulco nasolabial profundo, e ainda, má postura corporal com ombros caídos e voltados para frente). Estas crianças foram encaminhadas ao Serviço de Atendimento Otorrinolaringológico para tratamento.

O hábito de sucção provoca má postura da língua, mordida aberta e dentalização dos fonemas /t/, /d/,/s/,/z/, /l/ ${ }^{8}$. Além disso, o hábito de sucção de chupeta e mamadeira que até os 3 anos é nutricional, a partir desta idade se torna vicioso e deletério ${ }^{10}$. O hábito retirado aos 3 anos restabelece o equilibrio do sistema estomatognático. Foi observado também, que retirado o hábito precocemente normaliza-se a oclusão da criança na dependência do grau de deformidade ${ }^{2,9,10}$. Portanto, baseados na nossa amostra e na literatura, sugerimos a remoção do hábito da sucção vicioso através da eliminação dos fatores etiológicos desses hábitos (fatores emocionais e nutricionais). Ficou evidente que a resposta da criança frente ao distanciamento da mãe que se encontra no mercado de trabalho, manifesta-sepor meio de hábitos regredidos com mecanismos de compensação às sensações de insegurança, fato também observado em outra pesquisa ${ }^{7}$.

A intervenção ortodôntica deve ser iniciada com procedimentos preventivos, tais como: remoção do hábito, orientação familiar e, após os 6 anos de idade, interceptação das conseqüências destes hábitos no desenvolvimento da oclusão através de aparelhos ortodônticos removíveis ${ }^{16,19,22}$. Segundo alguns autores, a intervenção mecânica ortodôntica deve ocorrer somente após os 6 anos de idade ${ }^{4,22}$. Entretanto, somente a abordagem multidisciplinar da mordida aberta causada pelo hábito de sucção determina o sucesso do tratamento, sendo essencial uma visão panorâmica do tratamento, com a participação do ortodontista, do psicólogo, do otorrrinolaringologista e do fonoaudiólogo, para uma terapia eficaz e diminuição do risco de recidiva desta maloclusão.

Quanto às alterações fonoaudiológicas, notamos que a maior incidência foi o distúrbio articulatório $(55,55 \%)$, seguido por distúrbio da motricidade oral (33,33\%). Entretanto, notamos que hábitos orais viciosos podem causar alterações de tônus, de mobilidade de órgãos fonoarticulatórios, da respiração, da mordida, levando à alterações da fala, coincidindo com dados de outro estudo ${ }^{21}$. Salienta-se a necessidade da atuação da fonoterapia, quando distúrbios fonoaudiológicos decorrentes de hábitos orais de sucção estejam presentes. A fonoterapia atua na modificação do comportamento inadequado, tanto na postura dos lábios, língua, bochechas e mandíbula, ainda na coordenação do tipo respiratório, buscando conscientizar a criança e a famí- 
lia sobre a importância do hábito inadequado.

Foi constatado que as estruturas faciais em desenvolvimento na infância não seguem um padrão de crescimento imutável, mas um padrão que se altera pela aplicação de forças que variam a relação entre ossos e músculos. Estas forças podem ser provenientes de hábitos viciosos que alteram o sistema estomatognático. Os hábitos orais viciosos são certamente responsáveis pelo surgimento de diversos problemas como fonação atípica e maloclusões, como salientados nos nossos resultados e na literatura ${ }^{2,4,5}$. Ressaltase, assim, a necessidade de ações de promoção de saúde, com a participação interdisciplinar da Otorrinolaringologia, da Fonoaudiologia e da Odontologia para a prevenção de distúrbios ocasionados pelo hábito de sucção, bem como o tratamento reabilitador destes distúrbios, quando instalados, para a população de baixa renda, em serviços públicos.

\section{CONCLUSÕES}

Os hábitos orais de sucção foram fatores etiológicos de maloclusões e distúrbios fonoaudiológicos. A participação da otorrinolaringologia é fundamental na compreensão e evolução dos casos, nos quais há distúrbio relacionado às alterações anátomo-funcionais dos órgãos fonoarticulatórios e presença de hábitos orais viciosos. Assim, o acesso às especialidades envolvidas no tratamento dessas disfunções devem estar disponíveis em serviços públicos para a população de baixa renda.

\section{REFERÊNCIAS BIBLIOGRÁFICAS}

1. Gomes IVD, Proença MG, Limongi SCO. Temas em Fonoaudiologia. $5^{a}$ ed. São Paulo: Ed. Loyola; 1989. p.59-119.

2. Soares CAS, Totti JIS. Hábitos deletérios e suas conseqüências. Rev CROMG 1996;2:21-6.

3. Freud S. Oral habits. In: Obras Completas. Madrid, Espana: Ed. Nueva; 1973;T.3:3379-423.

4. Massler M. Oral habits: development and management. J Pedodontics 1983;7:109-19.

5. Henriques JFC, Janson G, Almeida RR, Dainesi EA, Hayasaki SM. Mordida aberta anterior: a importância da abordagem multidisciplinar e considerações sobre etiologia, diagnóstico e tratamento. Apresentação de um caso clínico. Rev Dent Press Ortod Orto Facial 2000;5:29-36.
6. Serra-Negra JMC, Pordeus IA, Rocha Jr JF. Estudo da associação entre aleitamento, hábitos bucais e maloclusões. Rev Odont USP 1997;11(2): 79-86.

7. Tomita NE, Sheiham A, Bijella VT, Franco LJ. Relação entre determinantes sócio-econômicos e hábitos bucais de risco para más-oclusões em pré-escolares. Pesq Odont Bras 2000;14: 16975 .

8. Wadsworth SD, Maul CA, Stevens EJ. The prevalence of orofacial myofunctional disorders among children identified with speech and language disorders ingrades kindergarten through six. Int J Orofac Myol 1998;24:1-19.

9. Alves AC, Bastos E. Hábito vicioso de sucção. Rev ABO Nac 1995;3:225-58.

10. Bayardo RE, Mejla JJ, Orozco SLE, Montoya KB.S. Etiology of Oral Habits. J Dent Child 1996;63(5):350-53.

11. Cordasco G. Bad Habits and dysgnathia epidemiological study. Stomatol Mediterr 1989;9(2):173-77.

12. Turgeons B, Lachapelle D. Nutritive and nonnutritive sucking habits: a review. J Dent Child 1996;63(5):321-27.

13. Tomita NE, Vitoriano TB, Franco LJ. Relação entre hábitos bucais e má oclusão em pré-escolares. Rev Pub Saúde 2000;34(3):299-303.

14. Chan C, Pinto AS, Martins JCR, Mendes AJD, Sakima PRT. Estudo cefalométrico dos efeitos esqueléticos e dentários do hábito persistente de sucção de chupeta. Rev Odont UNESP 1996;25:17182 .

15. Martins JCR, Sinimbú CMB, Dinelli TC, Martins, LPM Rauelli, DB. Prevalência de má oclusão em pré-escolares de Araraquara: relação da dentição decídua com hábitos e nível sócioeconômico. Rev Dent Press Ortod Ortop Facial 1998;3(6):543.

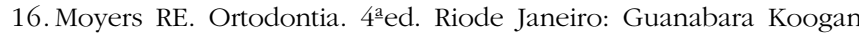
991;127(155):438-9.

17. Khalil AM. Short and long-term effects of thumb-rucking habit breaking appliance on speech in children. Egypt Dent J 1994;40(3):827-32.

18. Spinelli VP, Massari IC, Trenche MCB. Temas em fonoaudiologia. $5^{\underline{a}}$ ed. São Paulo: Ed. Loyola; 1989. p.122-97.

19. Popovich F, Thompson GW. Evaluation of preventive and interceptive orthodontic treatment between three end eighteen years of age. In: Transactions of the Third Internacional Orthodontic Congress. St. Louis: CV Mosby; 1975.

20. Hale ST, Kellum GD, Bishop FW. Prevalence of oral muscle and speech differences in orthodontic patients. Int J Orofac Myol 1988;14(3): 14-29.

21. Santos LK, Ávila CRB, Cechella C, Morais ZR. Ocorrência de alterações de fala do sistema sensoriomotor oral e de hábitos orais em crianças pré-escolares da primeira série do primeiro grau. Pro-fono 2000;12(2):93-101.

22. Mercadante MMN, Ferreira AFV. Ortodontia - diagnóstico e planejamento clínico. 3ํㅡㄹ $\mathrm{ed}$. Rio de Janeiro: Artes Médicas; 1986. p.246-270. 